YONGBIN YANG ${ }^{1}$

E-mail: 17120919@bjtu.edu.cn

PENG DU, Ph.D. ${ }^{2}$

(Corresponding author)

E-mail:pdu@bjtu.edu.cn

${ }^{1}$ China Railway Engineering Design

and Consulting Group Co., Ltd.

15 Guang'an Road, Fengtai District, Beijing 100055,

China

${ }^{2}$ School of Traffic and Transportation

Beijing Jiaotong University

Shangyuan village, Haidian District, Beijing 100044,

China
Traffic Planning

Original Scientific Paper

Submitted: 18 May 2020

Accepted: 26 Oct. 2020.

\title{
OPTIMIZATION OF THE SUBURBAN RAILWAY TRAIN OPERATION PLAN BASED ON THE ZONAL MODE
}

\begin{abstract}
Traditional all-stop train operation mode cannot meet the demand of long travel distance and centralized travel of commuters very well. To meet this special travel demand, a zonal train operation mode based on "many-to-many" train stops is proposed. The coefficient of passenger exchange is used to locate suburban areas by depicting travel characteristics of commuters. Operational separating points within the suburban area are used as decision variables to analyze the combined cost components of this model, including passenger travel costs and railway operating costs. An integer programming model with the lowest overall cost is established, and the genetic algorithm is employed to solve it. The results proved good relative benefits in operation costs and travel time. And the sensitivity analysis of both coefficient of passenger exchange and passenger intensity has shown that the zonal operation mode is suitable for suburban railways with centralized travelers. However, the research also shows that when the passenger volume rose to a very high level, the number of zones would be limited by the maximized capacity of railway lines, which may cause the decline of the relative operational efficien$c y$.
\end{abstract}

\section{KEYWORDS}

suburban railway; centripetal travel; train stop plan; zonal operation mode; integer program.

\section{INTRODUCTION}

The suburban railway takes the main transportation tasks of commuting passengers between the central city and suburban areas. Compared with urban subway passengers, suburban commuters have the characteristics of long travel distance and centripetal travel direction. The operation of suburban railway, if in conventional all-stop mode, will bring about a large amount of unnecessary stoppage time and thus become unbearable for long distance commuters. In order to improve the efficiency of commuting travel in large cities, suburban railway operators are expected to provide accelerated train services, without stopping at every station. Therefore, the appropriate design of train stop pattern, together with frequency, becomes a major focus of suburban railway operators and researchers.

From the perspective of train stop optimization, some scholars have studied the suburban transportation organization models aside from the conventional all-stop subway model [1]. In general, five kinds of train stop plans are commonly seen in reality, which are the all-stop operation, skip-stop operation, zonal operation, express/local operation, and combined stop operation [2]. Vuchic [3] and Shang [4] proposed methods to evaluate these stop strategies by summarizing, analyzing, and comparing each of them. Obviously, the all-stop strategy is the simplest stop pattern for operation since all trains have the same stop plan, and this strategy is still widely used in many cities [5]. However, this strategy unavoidably increases the total travel time for long-distance passengers due to the large number of stops during their journeys. Hence, the other four stop strategies have been emphasized in recent studies, especially the skip-stop operation strategy. A skip-stop policy involves trains operating on the same track by alternatively skipping certain stations to save time and increase operating speed [8]-[10]. Yang [11] proposed mathematical models for optimizing the skip-stop operation strategy in urban rail transit systems by considering different 
realistic conditions and also designed an efficient genetic algorithm to search for near-optimal solutions. Jamili [12] proposed the model for skip-stop patterns in urban transport systems and designed heuristic algorithms to obtain a robust solution. Though skip-stop operation has the advantages of relatively lower investment and ease of implementation, for suburban railways where the number of passengers decreases in one direction, this mode cannot reduce the running cost flexibly. In other words, it is more suitable for urban lines with balanced traffic than for suburban railways. For the express/local operation strategy, Xiong [6] and Gao [7] formulated a linear programming model to determine the stop stations for express trains with the purpose of maximizing the reduction in passenger travel time by express trains and at the same time minimizing the accordingly increased travel time for slow train passengers. The express/local operation strategy can provide different frequencies of transport services for different levels of stations, so as to ensure the benefits of the majority. However, it has a great loss of line throughput.

Suburban commuters usually gather along the vast suburban areas of the city beside the railway stations and arrive at several major stations or transfer stations in the central city which has significant centripetal travel characteristics in space, that is, "many-to-one". Centripetal travel in Tokyo and Shanghai accounted for $64 \%$ and $62.98 \%$ [13] respectively, which should attract enough attention in transportation organization.

Salzborn [14] introduced the meaning of the zonal stop mode for the first time in 1969. Wang [15] gave an optimal strategy of elevator group zoning dispatching in high-rise office buildings. The method is also applicable to bus scheduling at peak hours. The passengers in the two methods share the common characteristics of centripetal travel direction and lower exchange rate between stations on the way. That is, the coefficient of passenger exchange is lower [3], [16]. Goneimm [17] proposed to divide train service districts according to the centripetal characteristics of commuters. In this way, the "many-to-one" OD mode was adopted to improve the efficiency of transportation. At the same time, the mathematical model of quantitative analysis was established. The method is based on the demand characteristics of commuters and has a strong innovative significance. However, it is limited in the case where the central station is unique. In order to avoid excessive transfers at a single station, more and more cities have expanded the number of urban stations on suburban railways. At this time, a railway operation plan that can be applied to more complex and oriented "many-to-many" transportation needs to be proposed urgently.

From the above analysis, it can be seen that the key issue in zonal research is to determine the demarcation point, that is, to define the boundary between the urban area and the suburbs, and to group stations in the same area. In addition, it is necessary to consider whether the plan is feasible in terms of line capacity and what the operating benefits will be. To solve these problems, based on the characteristics of passenger exchange, we divide the suburban railway into suburban and urban areas. In the urban area, commuter trains operate as regular subways. In the suburban area, the train adopts the zonal mode, only staying at the corresponding section and boundary stations. Furthermore, we set up a mathematical model to describe the changes brought about in this way.

The rest of the paper is organized as follows: Section 2 introduces the travel characteristics of commuters and constructs a link between the passenger flow centrality and the zonal operation mode. Section 3 analyzes some of the basic conditions for building a mathematical model, including the method of transit assignment and the calculation of line passing capability. Section 4 establishes a mathematical model with zonal nodes as decision variables, and designs genetic algorithm solutions in Section 5. Section 6 verifies the feasibility of the model and algorithm through case calculation, and analyzes the range of passenger flow applicable to the method. Finally, we made conclusions in Section 7.

\section{TRAVEL CHARACTERISTICS AND OPERATION METHODS}

The urban subway stations are surrounded by various functional areas of the city, which can bring about lots of exchange needs. Correspondingly, suburban railways mainly serve commuting needs, and their stations are commonly surrounded by residential areas. This results in a relatively simple nature of the passengers in the suburbs and a low exchange demand. To verify this phenomenon, this section uses the coefficient of passenger exchange $(C P E)$ to describe the two types of passenger behavior. 


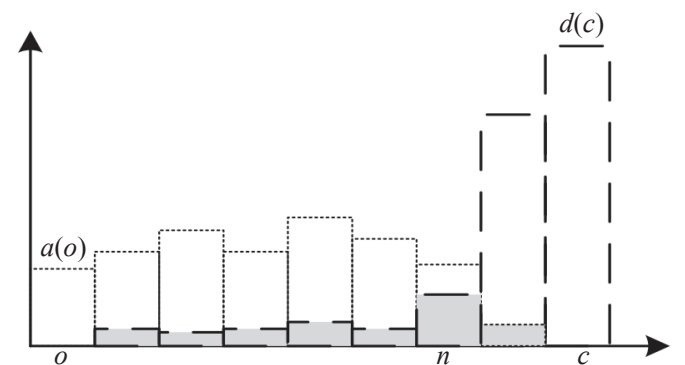

a)

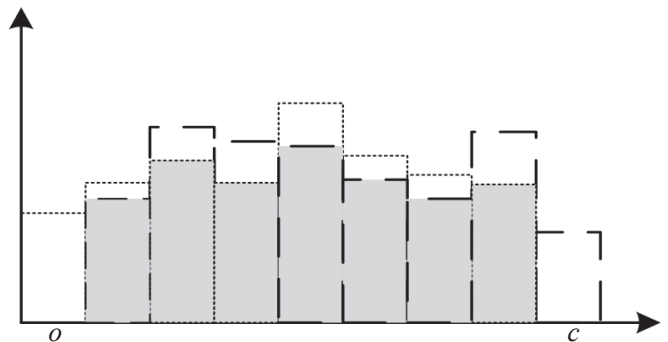

b)

-......... Boarding volume $\square$ Alighting volume

Figure 1 - Concept of CPE

Vuchic [3] defines $C P E$ as the ratio of total passengers who board along a line to those who are not replaced by alighting passengers. It indicates what portion of passengers are exchanged along a line, i.e., their turnover rate, which may reflect the characteristics of the travel process of the centripetal passenger, as shown in Equation 1. In it, $a(i)$ and $d(i)$ are the intensity of the passengers on and off each station (person/h) as shown in Figure 1. Each station accumulates the total number of passengers on board and those disembarked. The overlapping portion indicates the number of boarding passengers replaced by those getting off. $A_{I}$ and $D_{I}$ can be obtained by counting numbers on board and off board from all stations. The overlap part $P_{x}$ indicates the number of passengers on board replaced by those off board, that is, $A_{I} \cap D_{I}$.

$\eta_{x}=\frac{A_{I}}{A_{I}-P_{x}}$

Figure la shows the boarding and alighting situation of suburban railway passengers during morning commuting period. A large number of passengers boards the trains between stations $o$ to $n$, and further from station $n$ is the urban city area or the network hub station, where the passengers are centralized to get off or exchange. Passenger flow presents a many-to-many pattern in direction and quantity. The overlapping area $P_{x}$ in the figure, that is, the amount of passenger flow exchange along the way is very small, so that $\eta_{x}$ is also close to 1 . Correspondingly, Figure $1 b$ reflects the passengers boarding and alighting in a conventional subway line. Due to the diversity of land distribution along the subway line, the direction of passenger flow is chaotic and complicated, which presents an all-to-all pattern and does not show obvious regularity. The overlapping area $P_{x}$ is always at a high level, and the $C P E \eta_{x}$ is far greater than that of the suburban railway with the same passenger intensity.
According to the single-peak travel characteristics of suburban railway commuters, the stations along the line can be divided by critical station $n$ into two sections, each consisting one or several stations.

Suburban railway construction rarely ends at the edge of the city. On the line, there are usually multiple stations which have complex passenger characteristics similar to subway passengers. The "many-to-one" operation mode proposed by Ghoneim [17] is not suitable for most lines in this context. As opposed to this mode, the method proposed by this paper has a more practical application value in dividing the suburban section according to the nature of passenger travel.

In the "many-to-many" mode proposed in this paper, the mileage between $n-c$ stations in the urban area is short enough so that trains can be operated on regular subway mode. In the $o-n$ section where most of the lines are located in the suburbs, it is necessary to organize centripetal passengers in this area by special transportation methods.

\section{MODELING BASIS}

Based on the description of the suburban railway transportation mode and application scope, this section first briefly describes the problem. Next, the composition of the passenger flow at each station is analyzed. Finally, the line capacity is calculated to verify the feasibility of the model implementation in railway lines. These provide the basis for the establishment of mathematical models for the next section.

To facilitate modeling, the following assumptions need to be made first:

Hypothesis 1: The preference sequence of commuters for travel is accessibility, comfort, and non-transfer. When suburban trains are operated by means of 
mass transit, the passenger flow allocation scheme will be generated by the spontaneous choice of passengers rather than the strategy controlled by the ticket sales. The analysis of passenger behavior will have an impact on the modeling of train operation mode, and it is necessary to make realistic assumptions. Once the basic travel demand could be met, commuters with long travel distances would expect travel comfort and a departure train with seat.

Hypothesis 2: The suburban railway adopts a regular periodic schedule published to the public. Since the departure interval of suburban trains occupies the middle ground between subway and trunk railway trains, in order to facilitate trip planning and enhance service for passengers, the train schedule should be announced to the public. Studies have also shown that public timetables on suburban railways can affect the travel behavior of passengers and further affect modeling [18], [19].

Hypothesis 3: The vehicle scheduling of each running route during the study period is independent, and the stop plan in the up and down directions should be the same. The range of train stops in the operation plan will have an impact on the cost of vehicle scheduling, and the assumption can simplify the turnover process of the vehicle scheduling.

Based on the above three assumptions, model parameters are constructed and represented in Figure 2. $S=\{i \mid i=[1, n]\}$ is the station set of the suburban line section, and it is also the implementation scope of the zonal operation mode determined by the characteristics of CPE. $Y_{i}$ is the decision variable of the model used for judgment on whether station, $i$ is a zonal demarcation station, $Y_{i}=\{0,1\}, i \in S$. $B=\left\{b_{t-1} \mid t=[1, m+1]\right\}$ is a collection of sequences within the line, where $B \in S$ and $m=\sum_{i=2}^{n-1} Y_{i}$. If there are $\mathrm{m}$ demarcation stations in the suburbs, they can be divided into $m+1$ operation zones or train routing. The train collection is recorded as $T=\{t \mid t=[1, m+1]\}$.

The train operation process can be described in the following way: train $t$ originates from station $b_{t-1}$ and stops between station $b_{t-1}$ and $b_{t}$. And then train $t$ only stops at the transfer stations between station

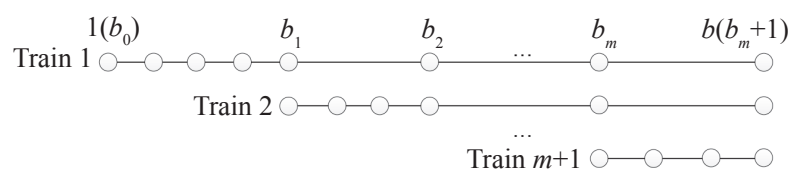

Figure 2 - Schematic diagram of the zonal operation mode $b_{t}$ and $n$ (it could be considered as station $b_{m+1}$ ). After station $n$, train $t$ arrives in the urban area, and all stops should be served.

To verify the feasibility of the zonal operation strategy, the diagram method is used to calculate the throughput of the timetable, as shown in Figure 3. st is the average stop time of a train. $I$ is the interval time of tracking train passing through the section.

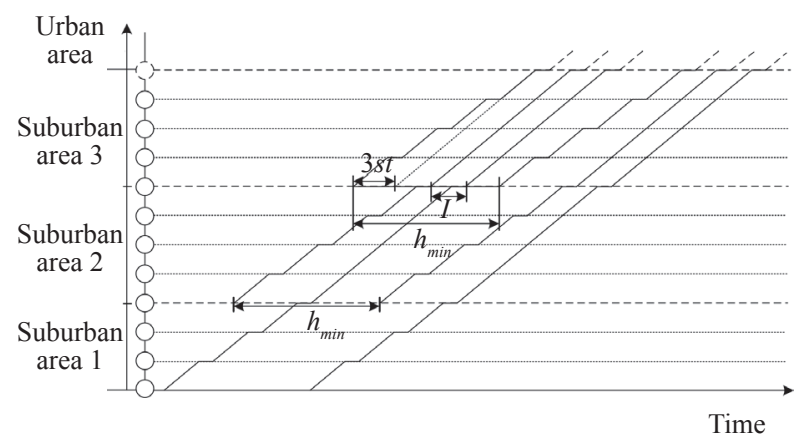

Figure 3 - Diagram of the passing through capacity of the zonal operation mode

Figure 3 shows the timetable of some trains in the morning peak. Most of the stations on the line are located in the suburbs. Suburban lines are divided into three operation zones, each of which operates departure commuter trains. The train operation line gradually becomes denser in the urban direction. Due to the presence of heterogeneous trains in the urban direction, the capacity of the timetable is reduced. When the trains are grouped, it can be seen that the urban-close region or the maximum heterogeneous region will be the bottleneck of the timetable capacity. The maximum operating frequency of all kinds of trains is $60 / h_{\min }$, and the minimum departure interval of the same train $h_{\text {min }}$ is shown in Formula 2.

$h_{\min } \geq \begin{cases}I \\ \max \left\{t I+s t\left(\sum_{i=b_{t-1}}^{b_{t}} x_{i}^{t}-2\right)\right\}, & t=2, \ldots, m+1\end{cases}$

The decision variable $Y_{i}$ can be used to determine the location of the demarcation station in the line, as well as the types of trains and other relevant parameters. The derived parameters proposed in this section can simplify the model.

For station $i$ :

$Z_{i}$-Operation zone corresponding to station $i$.

$t_{i}-$ Zonal trains - corresponding to station $i$.

$f_{i}$ - The service frequency of the train corresponding to station $i$. According to assumption $1, f_{i}=f_{t}, t \in t_{i}$. 
For train $t$ :

$X_{t} \quad-$ Stop stations set at the suburban section for train $t, X_{t}=\left\{x_{i}^{t} i[1, n], x_{i}^{t}=\{0,1\}\right\}$.

$Z_{t} \quad-$ Stations in the service zone of train $t$, $Z_{t}=\left\{i \mid i \in\left[b_{t-1}, b_{t}-1\right]\right\}$.

$Q_{i j}^{t} \quad$ - The OD statistics of train $t$ is obtained by adjusting the initial figure in Table 1.

$C_{\text {max }}^{t}$ - The maximum section passenger flow of train $t$, which is calculated by $Q_{i j}^{t}$.

$f_{t} \quad$ - Period operating frequency of train $t$.

\section{MODEL CONSTRUCTION}

According to the above-mentioned centripetal and low-exchange characteristics of commuters, if the suburban section is dismantled into several operating zones, the consumption of train stops on the way can be reduced and commuting speed of each zone can be improved. However, it may reduce the passing through capacity and increase transfer costs. According to the operation rules proposed in this paper, in this section the impact of the zonal mode for the passengers and enterprises is analyzed and mathematical models are established.

\section{Passenger travel cost}

According to the travel process for commuters, it can be seen that the passenger travel cost $C P$ is composed of three parts, as shown in Equation 3. $C W$, $C V$, and $C T$ represent the waiting cost, transit time cost, and transfer cost, respectively.

$$
C P=C W+C V+C T
$$

The waiting passengers include two types, waiting for the first ride or the transfer. The arriving behavior of the first ride passengers is related to the train departure interval and their travel habits, which is statistically subject to the $\beta$-uniform distribution [18], [19]. The relationship between the passenger waiting time and the train service frequency is shown in Equation 4. Transfer waiting time is related to the convenience of the transfer facility and the train connection interval, and can be considered part of the transfer cost.

Set unit passenger waiting cost as $\varepsilon_{t w}$ yuan/(person $\mathrm{min}$ ), and the total waiting time for passengers is shown in Equation 5.

$$
\begin{aligned}
& t w_{i}=t w\left(f_{i}\right) \\
& C W=\varepsilon_{t w} \sum_{i=1}^{n-1} \sum_{j=i+1}^{n} Q_{i j} t w\left(f_{i}\right)
\end{aligned}
$$

According to the train operation status, the transit time cost is composed of the section operating time and stopover time. Determined by the train traction and railway line conditions, the section operating time constitutes a fixed cost. This paper only takes variable operating cost into consideration, that is, the cost of passengers in transit for the stopover station, as shown in Equation 6.

$$
C V=\varepsilon_{t v} \sum_{t=1}^{m+1} \sum_{i=b_{t-1}}^{b_{t-1}} \sum_{j=i+1}^{n} Q_{i j}^{t} \sum_{i=i+1}^{j-1} x_{i}^{t}
$$

If stations $i$ and $j$ are not in the same operation zone and station $j$ is not a transfer station, according to Hypothesis 1 , passenger $Q_{i j}$ needs to transfer once. The average transfer cost is expressed as $\varepsilon_{t t}$, and the total transfer cost $C T$ is shown in Equation 7.

$$
C T=\varepsilon_{t t} \sum_{i=1}^{n-1} \sum_{j=i+1}^{n} Q_{i j}\left[1-\prod_{k=i+1}^{j-1}\left(1-Y_{k}\right)\right]\left(1-Y_{j}\right)
$$

Now we get Formula 8 ,

$$
\begin{aligned}
C P= & \varepsilon_{t w} \sum_{i=1}^{n-1} \sum_{j=i+1}^{n} Q_{i j} t w\left(f_{i}\right)+\varepsilon_{t v} \sum_{t=1}^{m+1} \sum_{i=b_{t-1}}^{b_{t-1}} \sum_{j=i+1}^{n} Q_{i j}^{t} \sum_{i=i+1}^{j-1} x_{i}^{t}+ \\
& +\varepsilon_{t t} \sum_{i=1}^{n-1} \sum_{j=i+1}^{n} Q_{i j}\left[1-\prod_{k=i+1}^{j-1}\left(1-Y_{k}\right)\right]\left(1-Y_{j}\right)
\end{aligned}
$$

After combination and simplification, Formula 9 is obtained.

$$
\begin{aligned}
C P & =\sum_{i=1}^{n-1} \sum_{j=i+1}^{n} Q_{i j}\left\{\varepsilon_{t w} t w\left(f_{i}\right)+\varepsilon_{t t}\left[1-\prod_{k=i+1}^{j-1}\left(1-Y_{k}\right)\right]\left(1-Y_{j}\right)\right\}+ \\
& +\varepsilon_{t v} \sum_{t=1}^{m+1} \sum_{i=b_{t-1}}^{b_{t-1}} \sum_{j=i+1}^{n} Q_{i j}^{t} \sum_{i=i+1}^{j-1} x_{i}^{t}
\end{aligned}
$$

\section{Operating costs}

According to Hypothesis 3, the up-and-down direction trains operate under the same schedule. So, the operating cost $C C$ consists of three parts: train travel cost $C L$, train start-stop cost $C P$, and average vehicle turnaround cost $C U$.

$$
C C=C L+C P+C U
$$

Since the trains in each operating zone are independent, the value of travel cost is the sum cost of each train routing, as shown in Equation $11 . l_{i}$ is the length of section $i, \beta_{i}$ is the running cost per unit mileage of the train, yuan $/ \mathrm{km}$.

$$
C L=\beta_{1} \sum_{t=1}^{m+1} \sum_{i=b_{t-1}}^{n-1} l_{i} f_{t}
$$

Energy consumption cost and station service cost for the train to start-stop at a station, yuan/station.

$C P=\beta_{2} \sum_{t=1}^{m+1} \sum_{i=b_{t-1}}^{n-1} x_{i}^{t} f_{t}$ 
The vehicle turnaround time decreases as the number of zones increases. However, due to the influence of the ceil rule on the calculation of train routing, the total number of trains may increase, as shown in Equation 13. Therefore, when determining the train operation mode, it is necessary to comprehensively consider the change in the average cost of vehicle turnaround, as shown in Equation 14.

$$
\begin{aligned}
& \sum_{t=1}^{m+1}\left\lceil\frac{C_{\max }^{t}}{C_{v}}\right\rceil \geq\left\lceil\sum_{t=1}^{m+1} \frac{C_{\max }^{t}}{C_{v}}\right\rceil \\
& C U=\frac{\beta_{3}}{30} \sum_{t=1}^{m+1} f_{t}\left[\sum_{i=b_{t-1}}^{n-1}\left(60 l_{i} \bar{\nu}^{-1}+x_{i}^{t} s t\right)+t_{T}\right]
\end{aligned}
$$

Among them, the car turnaround time includes the entire operating hours in the suburban and urban lines. The suburban operation is flexible enough so that its turnaround time should be counted in, whereas the running time in the urban operation will be counted as the other time. Then, the operating costs can be written as Formula 15.

$$
\begin{aligned}
C C= & \sum_{t=1}^{m+1} \sum_{i=b_{t-1}}^{n-1} f_{t}\left[\beta_{1} l_{i}+\beta_{2} x_{i}^{t}+\frac{\beta_{3}}{30}\left(60 l_{i} \bar{\nu}^{-1}+x_{i}^{t} s t\right)\right]+ \\
& +\frac{f_{t} \beta_{3} t_{T}}{30}
\end{aligned}
$$

\section{Constraints}

The train departure frequency $f_{t}$ should meet the needs of the largest passenger section in the $t$-route and does not exceed the passing through capacity which satisfies the relationship of Equations 16 and 17. $C_{v}$ is the train capacity, person/train.

$$
\begin{aligned}
& f_{t} \geq\left[\frac{C_{\max }^{t}}{C_{v}}\right], \quad t=1, \ldots, m+1 \\
& f_{t} \leq 60\left(t I+s t \sum_{i \in t} x_{i}^{t}\right)^{-1}, \quad t=1, \ldots, m+1
\end{aligned}
$$

In order to ensure that all travel demands during this time period can be satisfied within one transfer, the zonal demarcation station should be set as the mandatory stop for all trains, as shown in Equations 18 and 19.

$$
\begin{aligned}
& (m+1) Y_{i} \leq \sum_{t=1}^{m+1} x_{i}^{t}, i=b_{t-1}, \ldots, n \\
& Y_{1}=Y_{n}=1
\end{aligned}
$$

\section{Optimization model}

Since all the costs have been unified into the economic unit: yuan, the generalized comprehensive cost of the operation mode can be expressed by Equation 20.

$$
\begin{aligned}
& \min Z=c c+C P \\
& \text { s.t. expressions }(16) \sim(19)
\end{aligned}
$$

\section{ALGORITHM DESIGN}

Dynamic programming algorithm and genetic algorithm have been widely used to solve the problem of elevator stop in different zones and a similar research by Ghoneim [17] has been applied to suburban transportation. Wang [13] compared two algorithms in the case of elevator zoning, and proved that the genetic algorithm has higher speed when the alternative scale is small, which is suitable for solving suburban railway trouble within fewer stations. The scale of the solution with $n$ stations is $2^{n-2}$, and the solution scale is relatively small. Therefore, the genetic algorithm is designed to solve the main process as follows:

1) According to passenger travel characteristics, we define the station range of the zonal operation mode.

2) Encoding the chromosome in binary mode. Each chromosome is a $0-1$ array of 1 row and $n$ columns, which is marked as 1 by the demarcation station and 1 is the first column and the nth column. If not, it is marked as 0 .

3) Corresponding to the initial population, the $\mathrm{OD}$ statistics is allocated by the method of table 1 , and $Q_{i j}^{t}$ of each train is generated.

Table 1 - Passenger flow composition of train $t$

\begin{tabular}{||l|c|c||}
\hline \multicolumn{1}{|c|}{ From/To } & $\begin{array}{c}\text { Present } \\
\text { section } Z_{t}\end{array}$ & $\begin{array}{c}\text { The previous } \\
\text { station }\end{array}$ \\
\hline \hline Passed station $\left[1, b_{t-1}-1\right]$ & $\sqrt{ }$ & \\
\hline This section $Z_{t}$ & $\sqrt{ }$ & $\sqrt{ }$ \\
\hline
\end{tabular}

According to Hypothesis 1, all trains need to stop at the boundary station for passengers to transfer. And the passengers departing from the boundary station prefer the empty car from originating station. Then the composition of the passenger flow in train $t$ can be obtained by adjusting the initial OD data according to the stop plan, as shown in Table 1.

4) According to the results of the OD statistics, the operation cost of trains in each mode is calculated, and the constraints are incorporated into the objective function as penalty terms. Then the results of the objective function are converted into the fitness function. Define evaluatePopulation(population[generation]) $=$ sum(objectiveValue(individual)) + penaltyCoefficient . sum(penaltyValue); 
5) Before the termination condition is reached, the individuals with higher adaptability are selected by roulette rules, and the offspring is generated by crossing and mutation; then the process of steps $2-4$ is repeated.

In order to facilitate the understanding of the algorithm process, a pseudocode could be listed.

generation $=0$;

population [generation] = initializePopulation(populationSize); evaluatePopulation(population[generation]);

While isTerminationConditionMet() $==$ false do parents $=$ selectParents(population[generation]);

population[generation +1$]=$ crossover(parents);

population[generation +1$]=$ mutate(population[generation +1$]$ );

evaluatePopulation(population[generation]);

generation ++ ;

End loop;

\section{CASE ANALYSIS}

In this section, the rationality of the model and algorithm design is verified by an example. On this basis, sensitivity analysis of the two main parameters is carried out to further explain the applicable conditions of the zonal operation mode.

\subsection{Parameter input}

There are 14 stations in a suburban railway, among which the $12^{\text {th }}, 13^{\text {th }}$, and $14^{\text {th }}$ station are located in the downtown area. The $1^{\text {st }}$ to $11^{\text {th }}$ station are located in the suburban whose boarding volumes are larger than the alighting ones. According to that, a zonal operation mode is established between stations $1-12$ of the line, and the OD statistics in the morning commuting period (one hour) of the case line is shown in Table 2.

It can be seen from the data in Table 2 and Figure 4 that the passenger volume in this period is large and the main direction points to the central city. The closer to the city center, the greater the passenger volume is. Lots of people boarding the train between stations 1 to 11 and alighting it after station 12. The CPE between stations 1-12 is 1.13 , and the exchange passengers only account for $11.5 \%$ of the total travel volume. We take it as an example to design a zonal operation mode for commuting direction in the morning commuting period. The values of the parameters are shown in Table 3.

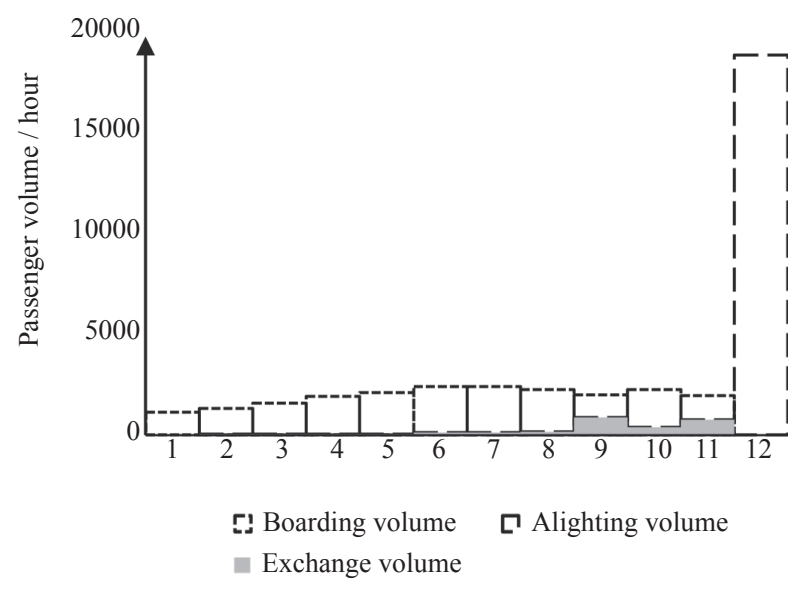

Figure 4-Passenger exchange situation of the case line

Table $2-O D$ data in the morning commuting period of the case line

\begin{tabular}{||c|c|c|c|c|c|c|c|c|c|c|c|c||}
\hline \hline Station & 1 & 2 & 3 & 4 & 5 & 6 & 7 & 8 & 9 & 10 & 11 & 12 \\
\hline 1 & 0 & 6 & 11 & 10 & 13 & 19 & 14 & 16 & 67 & 22 & 64 & 904 \\
\hline 2 & 0 & 0 & 2 & 18 & 8 & 24 & 19 & 23 & 72 & 34 & 57 & 1,065 \\
\hline 3 & 0 & 0 & 0 & 13 & 10 & 25 & 14 & 23 & 91 & 29 & 54 & 1,348 \\
\hline 4 & 0 & 0 & 0 & 0 & 11 & 42 & 20 & 20 & 118 & 34 & 38 & 1,667 \\
\hline 5 & 0 & 0 & 0 & 0 & 0 & 31 & 21 & 21 & 139 & 45 & 52 & 1,826 \\
\hline 6 & 0 & 0 & 0 & 0 & 0 & 0 & 26 & 28 & 114 & 54 & 59 & 2,169 \\
\hline 7 & 0 & 0 & 0 & 0 & 0 & 0 & 0 & 26 & 177 & 40 & 98 & 2,092 \\
\hline 8 & 0 & 0 & 0 & 0 & 0 & 0 & 0 & 0 & 84 & 63 & 87 & 2,036 \\
\hline 9 & 0 & 0 & 0 & 0 & 0 & 0 & 0 & 0 & 0 & 51 & 62 & 1,891 \\
\hline 10 & 0 & 0 & 0 & 0 & 0 & 0 & 0 & 0 & 0 & 0 & 201 & 2,076 \\
\hline 11 & 0 & 0 & 0 & 0 & 0 & 0 & 0 & 0 & 0 & 0 & 0 & 1,985 \\
\hline 12 & 0 & 0 & 0 & 0 & 0 & 0 & 0 & 0 & 0 & 0 & 0 & 0 \\
\hline \hline
\end{tabular}


Yang Y, Du P. Optimization of the Suburban Railway Train Operation Plan Based on the Zonal Mode

Table 3 - Values of the parameters

\begin{tabular}{|c|c|c|c|c|c|c|c|}
\hline Parameter & Meaning & Value & Unit & Parameter & Meaning & Value & Unit \\
\hline$\varepsilon_{t w}$ & $\begin{array}{l}\text { Waiting } \\
\text { cost }\end{array}$ & 0.5 & yuan/(person·min) & $\bar{v}$ & $\begin{array}{l}\text { Average section running } \\
\text { speed }\end{array}$ & 60 & $\mathrm{~km} / \mathrm{h}$ \\
\hline$\varepsilon_{t v}$ & In-train time cost & 1 & yuan/(person·min) & $C_{v}$ & Train capacity & 1,322 & $\begin{array}{l}\text { person/ } \\
\text { train }\end{array}$ \\
\hline$\varepsilon_{t t}$ & Transfer cost & 5 & yuan/(person·min) & $I$ & $\begin{array}{l}\text { Minimum departure } \\
\text { interval }\end{array}$ & 2 & $\min$ \\
\hline$\beta_{1}$ & $\begin{array}{l}\text { Train running } \\
\text { cost }\end{array}$ & 100 & yuan/km & $t_{T}$ & Urban running time & 15 & $\min$ \\
\hline$\beta_{2}$ & $\begin{array}{l}\text { Train stop } \\
\text { cost }\end{array}$ & 450 & yuan/train stop & $\bar{l}_{i}$ & Average station spacing & 2 & $\mathrm{~km}$ \\
\hline$\beta_{3}$ & $\begin{array}{l}\text { Vehicle use } \\
\text { cost }\end{array}$ & 2000 & yuan/(train·h) & & & & \\
\hline
\end{tabular}

\subsection{Solution results}

Based on the algorithm, a Matlab program is designed to obtain the optimal operation mode as shown in Figure 5.

There are 4 types of trains running in the suburban railway sections, and the stop range covers the corresponding service zone and boundary transfer station. The form of feasible solution is consistent with our design, which proves the validity of the model. In this mode:

1) On route 1 , train stops are reduced to $1 / 3$ of the original plan, and the spatial and temporal accessibility of the outer suburban area is increased. Compared with the original mode, the attraction of the region to commuters will be improved.

2) Trains on route 2 and 3 attract the commuters in the middle distance, and the commuting time is partially compressed. The suburban areas covered by the trains on route 2 and 3 have a high comprehensive competitiveness in the commuting time and the living cost in the city which can reduce the cost of passengers and enterprises significantly.

3) On route 4, the trains attract commuters at the edge of the urban city and provide transfer services for passengers in the suburbs. Operation of a departure train in this zone can reduce passenger retention in the commuting periods and improve the efficiency of vehicle turnaround.

\subsection{Cost comparison}

Compared with the conventional operation mode of single routing, $29.45 \%$ of the comprehensive cost can be saved in this mode. The cost comparison between the two modes is shown in Table 4, and the following conclusions can be drawn from the analysis.

1) Compared with the conventional mode, the zonal mode provides differentiated services to stations along the line. Although the train service frequency of some stations is reduced, most trains

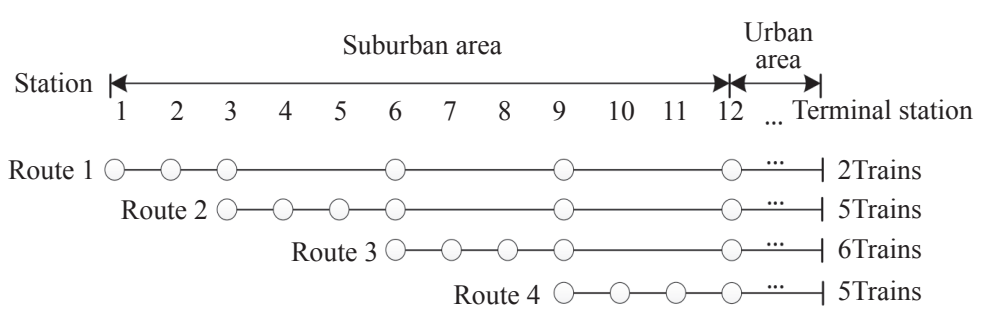

Figure 5 - Morning commuting period train operation mode

Table 4 - Cost comparison between two modes (unit: yuan/hour)

\begin{tabular}{||l|c|c|c|c|c|c|c||}
\hline & Cost CW & Cost CV & Cost CT & Cost CL & Cost CP & Cost CU & Total Cost \\
\hline \hline Zonal operation mode & 33,198 & 43,270 & 5,350 & 23,600 & 41,400 & 39,867 & 186,685 \\
\hline Conventional operation mode & 10,790 & 90,806 & 0 & 33,000 & 81,000 & 49,000 & 264,596 \\
\hline
\end{tabular}


improve their running speed significantly. When publishing the timetable, more commuters will arrive at the station on time instead of randomly, and then the waiting cost will not increase linearly by the departure interval.

2) Although the zonal mode adds the transfer process for the passengers, there are only a few passengers who travel short distances in the suburban area during the commuting period, and the increased transfer cost should not be significant.

3) According to Equation 13, the total number of trains may increase during this period. However, the mode improves the efficiency of the vehicle turnaround by diversifying the routes and reducing the stoppage. In turn, it reduces the operating costs and passenger congestion.

\subsection{Sensitivity analysis}

In this section, the sensitivity analysis of key parameters is carried out to verify the applicability of the zonal operation mode for passengers.

\section{Applicability analysis of $C P E$}

The paper qualitatively analyzes the relationship between the CPE and the zonal operation method, verifies the relative benefit of the mode through an example line with low $C P E$. However, to further clarify the logical relationship between the two factors, it is necessary to verify the sensitivity of passenger conditions.

Generally, the sensitivity analysis of parameters needs to keep other relevant parameters fixed. However, it is difficult to accomplish because of the change of the $C P E$ combined with the adjustment of the basic OD table and other independent variables. In order to reduce the influence of other variable floating on parameter analysis, we keep the total number of passengers unchanged and adjust the arrival distribution of OD statistics. In this way, we can still compare the relative operating benefits of different operation modes under different $\eta_{x}$, and keep the cost scale in a relatively stable range.

The comparison of the calculation results in Figure 6 shows the following:

1) The comprehensive cost of the conventional mode varies greatly with the change of the $C P E$, and the cost is reduced by about one third when $C P E$ increases from 1 to 2 . That is because the weaker the centrality of the commuters or the higher the $C P E$, the shorter the average passenger occupancy time of the train resources. The passenger volume in the section is reduced and fewer vehicles will be used. And the cost in transit is lower as the average passenger travel distance gets shorter. It shows the unique passenger characteristics for suburban railways.

2) The comprehensive cost of the zonal operation mode varies little with the change of the CPE. This is because the CPE increases as the average passenger distance decreases. At this time, the advantages of the zonal operation decrease and the comprehensive cost basically remains unchanged.

3) The relative benefit of the zonal operation mode for the cost savings of the conventional solution is continuously narrowed to 0 as the $C P E$ increases. This indicates a high correlation between the $C P E$ and the efficiency of the zonal operation mode. That is, the mode is applicable to the low $C P E$ suburban lines. When the $C P E$ is greater than 2 , the centrality of passengers is not obvious. At this time, the passenger flow assumed by the line is complex and zonal operation mode will not be generated.

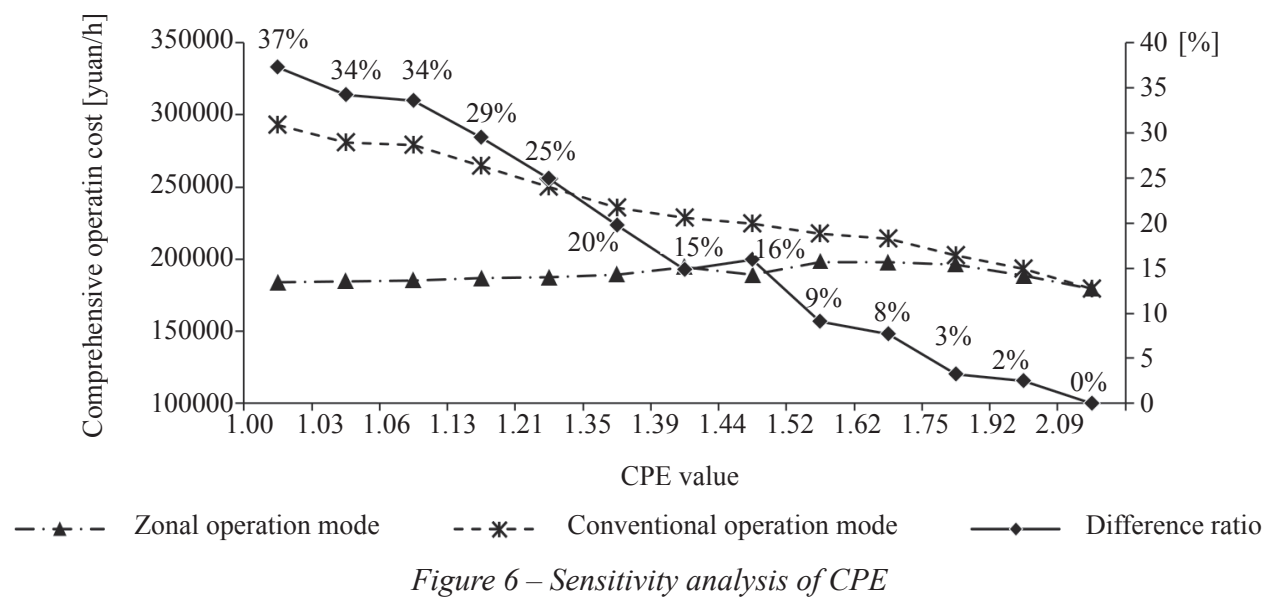


The conclusion proves that there is a high correlation between the CPE and the benefit of the zonal operation mode. This mode is obviously applicable to the low $C P E$ suburban commuter lines.

\section{Sensitivity analysis of the passenger flow intensity to the mode}

In section 2.5 , the analysis of the passing capacity of the zonal operation timetable suggests that the benefit improvement of the divisional operation mode is at the expense of the line capacity, and the magnitude improvement of the passenger volume may have an impact on the result of the mode. In order to verify the conclusion and further clarify the adaptive conditions of the mode, sensitivity analysis is carried out.

With the other parameters unchanged, the OD statistics in the case is scaled by $0.8-1.5$ times. Compare the change of operation benefits under the actual constraints and ideal conditions, as shown in Figure 6. In practical constraints, the optimal mode is limited by the line equipment conditions. In an ideal situation, the capacity of the zonal mode can be fully released by constructing the overtaking lines in stations or sections. The benefits of the two modes differ under the actual constraints.

It can be seen in Figure 7 that when the passenger volume floats between 0.8 and 1.3 times, the calculation results of the two modes are almost the same. This shows that when the line passing capacity is sufficient, the regional operation mode is more efficient. As the number of passengers continues to increase, the throughput of the line begins to be saturated. Solutions with the best operational benefits are available at this time. It is foreseeable that trains will be operated at the minimum interval to accomplish the maximum of passenger transportation volume.

In the face of high-intensity suburban passengers that are further attracted by the advanced service, the suburban railway operator in metropolis usually constructs a double track in the bottleneck stations or sections with limited capacity, so that the superior partitioning mode can be adopted. In reality, the four-track parallel mode is adopted in some sections of the Tobu Tojo Line in the Tokyo private railway, which eliminates the capacity bottleneck of trains of different speed grades and keeps a relative balance between transportation efficiency and reconstruction cost.

\section{Sensitivity analysis of the algorithm parameters}

In order to verify the accuracy and efficiency of setting parameter values in the algorithm solving process, we take the penalty coefficient of the infeasible condition as an example for the sensitivity analysis.

In the GA algorithm in this case, the population size is set to 50 , the genetic algebra is set to 20 , and the penalty coefficient of the infeasible condition is 10 to the power of $2-6$. For each case, 20 repeated experiments were performed and the probability of obtaining the optimal solution 186,685 after 20 generations of the genetic process was calculated.

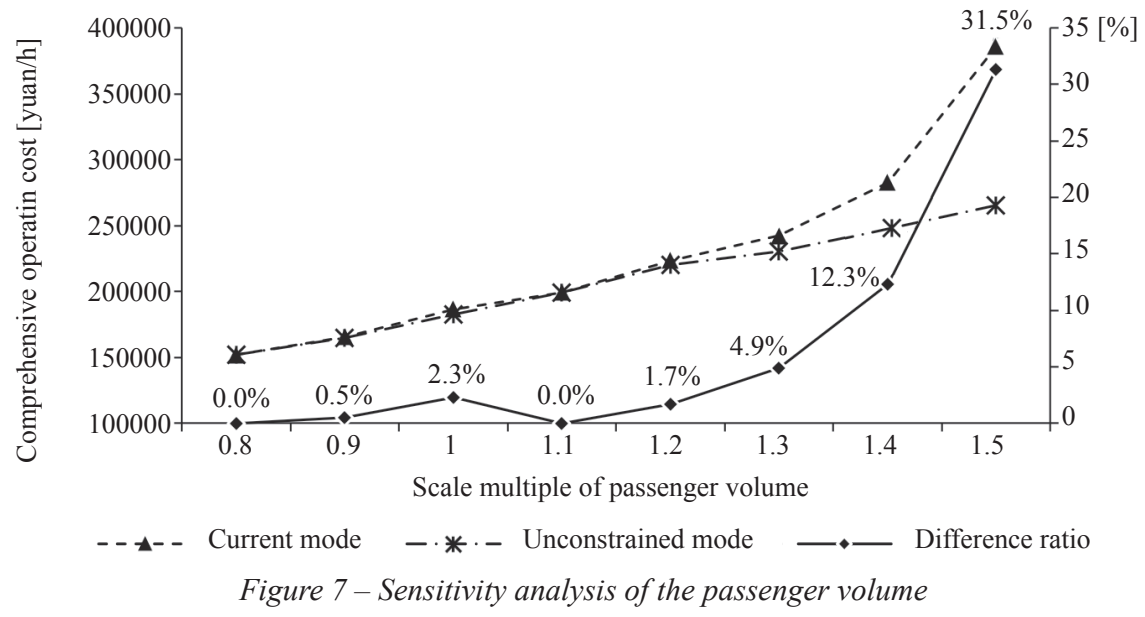

Table 5 - The best solution ratio under different penalty coefficients

\begin{tabular}{||c||c|c|c|c|c|c||}
\hline Penalty coefficient & 100 & 1,000 & 10,000 & 100,000 & $1,000,000$ & $10,000,000$ \\
\hline Best solution ratio & 0 & 0 & $55 \%$ & $80 \%$ & $70 \%$ & $65 \%$ \\
\hline
\end{tabular}


When the penalty coefficient is 100 or 1000 , the optimal solution, or even a feasible solution, cannot be obtained in multiple trials. This is because the penalty for infeasible conditions is too small, so that even if some conditions are not feasible, the fitness of the solution still seems to be the highest, thus becoming the optimal individual.

Once the penalty coefficient is greater than 10,000 , the target value of the infeasible solution plus the penalty value is no longer lower than 186,685 , and we can finally obtain the feasible optimal solution. It is worth noting that the penalty coefficient is not the larger, the better. A larger penalty coefficient greatly reduces the probability of infeasible solutions in the population, thereby reducing the diversity of the population and reducing the probability of obtaining the optimal solution within 20 generations. Therefore, we determined that using 100,000 as the penalty coefficient has the highest solution efficiency.

\section{CONCLUSIONS}

According to the characteristics of the commuters with high degree of centripetal travel, this paper systematically introduced the method and application of the modelling solution of the train operation plan with sub-regional stops.

First, it can be concluded that the many-to-many model is more adaptable than the many-to-one model. By considering the passenger exchange coefficient of the suburban railway, the paper proposes to use the zonal method for centripetal instead of all-to-all travel. And the classic many-to-one model is extended to many-to-many model, based on which a more adaptable optimization model is proposed and verified. Second, although the adoption of a zoning plan has a relative cost advantage within a certain range, the carrying capacity of the railway line cannot be neglected. When the intensity of the passenger flow continues to increase, the suburban railway authority needs to evaluate comprehensive costs and adjust the zoning operations or invest in the project to expand the capacity of the line, if necessary.

The passenger distribution model in the paper can be improved and the operational benefits also need to be verified by obtaining more data on the suburban railway lines. These issues need to be studied in future to improve the transportation theory and methods proposed in this paper.

\section{ACKNOWLEDGMENTS}

This research is supported by the National Natural Science Foundation of China (Grant No. 71971021).

杨雍涁 1

电子邮箱: 17120919@bjtu.edu.cn

杜鹏, 博士 ${ }^{2}$

（通讯作者）电子邮箱: pdu@bjtu.edu.cn

1 中铁工程设计咨询集团有限公司,

中国北京市丰台区广安路15号, 100055

2 北京交通大学交通运输学院,

中国北京市海淀区上园村3号, 100044

\section{基于分区域模式的市郊铁路列车开行方案优化}

\section{摘要}

传统的站站停列车运输模式难以较好地满足通 勤者的长距离、集中出行需求。针对这个问题, 提 出了基于 “多对多” 列车停站的分区域列车运行模 式。使用乘客换乘系数描述通勤者的出行特征, 并 被用作界定市郊区域的依据。接下来, 市郊区域内 的运营分界点被用作决策变量, 以分析在这种模式 下的综合成本构成, 包括乘客出行成本和企业运营 成本等。建立了总成本最低的整数规划模型, 并采 用遗传算法对其进行求解。结果表明, 研究方法在 运营成本和出行时间方面具有良好的相对效益。对 乘客换乘系数和客流强度的敏感性分析则表明, 分 区域运营模式适用于乘客集中出行的市郊通勤铁 路。同时研究还表明, 当客流强度上升到较高水平 时, 区域划分的数量将受线路通过能力限制, 从而 可能导致相对运营效益降低。

\section{关键词}

市郊铁路; 向心化出行; 列车停站方

案; 分区域运营模式; 整数规划

\section{REFERENCES}

[1] Qi J, Yang L, Di Z, et al. Integrated optimization for train operation zone and stop plan with passenger distributions. Transportation Research Part E: Logistics and Transportation Review. 2018;109: 151-173.

[2] Yang L, Qi J, Li S, et al. Collaborative optimization for train scheduling and train stop planning on high-speed railways. Omega. 2016;64: 57-76.

[3] Vuchic VR. Urban Transit: Operation, Planning and Economics. New Jersey, USA: John Wiley \& Sons, Inc.; 2005.

[4] Shang P, Li R, Yang L. Demand-driven timetable and stop pattern cooperative optimization on an urban rail transit line. Transportation Planning and Technology. 2020;43(1): 78-100.

[5] Yin J, Yang L, Tang T, et al. Dynamic passenger demand-oriented metro train scheduling with energy-efficiency and waiting time minimization: Mixed-integer linear programming approaches. Transportation Research Part B: Methodological. 2017;97: 182-213. 
[6] Xiong Y. Research on the express/slow train of the zonal rail line. Graduate thesis. Southwest Jiaotong University; 2012. p. 32-40.

[7] Gao Y, Yang L, Gao Z. Energy consumption and travel time analysis for metro lines with express/local mode. Transportation Research Part D. 2018;60: 7-27.

[8] Lee E, Lee I, Cho S, et al. A Travel Behavior-Based SkipStop Strategy Considering Train Choice Behaviors Based on Smartcard Data. Sustainability. 2019;11(10): 2791.

[9] Luo Q, Hou Y, Li W, et al. Stop Plan of Express and Local Train for Regional Rail Transit Line. Journal of Advanced Transportation. 2018;2018(3): 1-11.

[10] Wang Y, De S, Van D, Ning B. Efficient bi-level approach for urban rail transit operation with stop-skipping. IEEE Transactions on Intelligent Transportation Systems. 2014;15(6): 2658-2670.

[11] Yang A, Huang J, Wang B, et al. Train Scheduling for Minimizing the Total Travel Time with a Skip-stop Operation in Urban Rail Transit. IEEE Access. 2019;99(7): 81956-81968.

[12] Jamili A, Aghaee M. Robust stop-skipping patterns in urban railway operations under traffic alteration situation. Transportation Research Part C: Emerging Technologies. 2015;61: 63-74.
[13] $\mathrm{Xu}$ Z. Study on Job-Housing Relationship and Characteristic of Commuting in Shanghai: Based on the Perspective of Rail Transit Passenger Flow Data. Shanghai Urban Planning Review. 2016;(2): 114-121.

[14] Salzborn F. Timetables for a suburban rail transit system. Transportation Science. 1969;3: 279-316.

[15] Wang D, Li B. An Optimization Model of Elevators Group Zoning Dispatching and Its Application. CDEE. 2010;1: 18-21.

[16] Zhang X. Urban rail transit operation management. Beijing: Higher Education Press; 2017.

[17] Ghoneim N, Wirasinghe S. Optimum zone structure during peak periods for existing urban rail lines. Transportation Research Part B: Methodological. 1986;20(1): 7-18.

[18] Ingvardson J, Nielsen O, Raveau S, et al. Passenger arrival and waiting time distributions dependent on train service frequency and station characteristics: A smart card data analysis. Transportation Research Part C: Emerging Technologies. 2018;90: 292-306.

[19] Du P, Yang Y. Optimization of suburban train operation plan in mixed arrival mode. Journal of Transportation Systems Engineering and Information Technology. 2018;18(06): 92-98. 